\title{
Cenplestao
}

\section{O patrimônio cultural nas cidades inteligentes}

\author{
Juliana Martins de Castro \\ Doutoranda; Universidade Federal de Minas Gerais, Belo Horizonte, MG, Brasil; \\ julianamartins.castro@gmail.com \\ Renata Maria Abrantes Baracho \\ Doutora; Universidade Federal de Minas Gerais, Belo Horizonte, MG, Brasil; \\ renatabaracho@ufmg.br
}

\begin{abstract}
Resumo: $\mathrm{O}$ artigo tem como objetivo a investigação da temática da preservação patrimonial no contexto das cidades inteligentes. As smart cities, ou cidades inteligentes, constituem uma proposta relativamente recente, cujos primeiros estudos datam da década de 1990, e que começou a se difundir a partir da década de 2010. Essas propostas visam transformar o espaço urbano e seus serviços de modo "inteligente", com o uso das novas tecnologias da informação e comunicação (TICs), em vários campos de atuação, tais como saúde, mobilidade, segurança, dentre outros. Entretanto, observa-se nessas propostas uma abordagem quase inexistente a questões relacionadas ao patrimônio cultural, o que motivou o presente estudo. Para tanto, foi realizada uma revisão sistemática de literatura (RSL), contando com 56 publicações de diferentes países, conjunto que revela algumas questões importantes para o campo patrimonial frente a projetos de smartificação de cidades, como as que já vem ocorrendo no Brasil. Como conclusões, aponta-se a proposta desses estudos de constituição de uma nova forma de preservação patrimonial - o "patrimônio inteligente", discutindo suas implicações, limitações e possibilidades.
\end{abstract}

Palavras-chave: Patrimônio cultural. Preservação. Conservação. Cidades inteligentes. Patrimônio inteligente.

\section{Introdução}

Nas últimas décadas, o conceito de cidades inteligentes (ou smart cities) vem sendo discutido como uma das alternativas para o desenvolvimento ou remodelamento de áreas urbanas, devido a uma série de problemas ligados ao significativo aumento de sua população, à ineficiência de infraestruturas e serviços básicos e ao esgotamento de recursos naturais.

Alguns autores definem as cidades inteligentes como estratégias holísticas para mitigar os problemas urbanos, baseadas em planos, ações e decisões mediadas pelas novas tecnologias da informação e comunicação (TICs) 
em algumas áreas-chave da vida em sociedade, tais como economia, cidadania, governança, mobilidade, meio ambiente e qualidade de vida. Na prática, porém, a área tecnológica vem monopolizando os estudos e as práticas relacionadas às cidades inteligentes, o que pode ser percebido por meio de uma simples consulta às bases de dados de artigos científicos.

Como exemplo, a busca por "cidades inteligentes" no Google Acadêmico oferece aproximadamente 3.790 resultados, enquanto a busca por "cidades inteligentes" e "tecnologia" oferece 3.030 resultados, o que significa que quase $80 \%$ das publicações sobre cidades inteligentes, atualmente, contam com uma maior ou menor abordagem a aspectos tecnológicos. O mesmo, entretanto, não pode ser dito das outras áreas mencionadas na literatura: repetindo o experimento, a abordagem sobre a economia fica em cerca de $60 \%$ (2.360 resultados), sobre a mobilidade em aproximadamente 50\% (2.030 resultados), sobre a qualidade de vida e o meio ambiente em cerca de $45 \%$ cada (1.770 e 1.700 resultados, respectivamente), a governança em quase 30\% (1.120 resultados) e a cidadania em $23 \%$ das publicações ${ }^{1}$.

Outra dimensão que não é diretamente abordada entre esses estudos é a vida cultural da cidade, constituída por práticas, manifestações e equipamentos culturais, bem como pelos bens históricos patrimoniais. Os trabalhos acima mencionados tendem a incluí-la como um dos vários indicadores de mensuração das áreas cidadania ou qualidade de vida. A cultura, assim, constitui um subtópico para a análise das cidades inteligentes, e o patrimônio cultural um subtópico da cultura, o que explica que, juntos, esses dois assuntos estejam presentes em apenas $11 \%$ das publicações brasileiras sobre cidades inteligentes ${ }^{2}$.

Contudo, a cultura, enquanto "[...] um sistema de significados, atitudes e valores partilhados e as formas simbólicas [...] em que eles são expressos ou encarnados" (BURKE, 2010, p. 10), não somente é uma parte fundamental da vida coletiva, como viabiliza a própria existência das sociedades. Os modos como as pessoas de determinado lugar vivem, interagem, transitam, consomem e trabalham são parte dessa cultura, que têm muitos pontos em comum com diversos outros lugares ao redor do mundo, mas também apresentam especificidades que, por si só, já indicam a necessidade de se considerar a 
cultura, bem como o patrimônio cultural, para além do papel de um entre vários subtópicos em uma estratégia urbana.

Na medida em que os projetos ligados às cidades inteligentes vêm sendo desenvolvidos e aplicados em diversos locais, inclusive no Brasil, e a ênfase na utilização de novas tecnologias se mostra preponderante, surge uma dúvida para aqueles que se dedicam à preservação do patrimônio cultural das cidades: haveria uma preocupação, nesse modo de pensar o espaço urbano, com os bens que fazem parte da memória e da história da população? Existiria alguma diretriz "inteligente" para o patrimônio cultural?

Esses questionamentos constituem a base do presente estudo, que utiliza a metodologia de revisão sistemática de literatura (RSL) com o objetivo principal de compreender de que forma o patrimônio cultural é (ou não) contemplado nas tentativas de desenvolvimento das metodologias ligadas às cidades inteligentes. A análise do corpus documental selecionado oferece alguns caminhos interessantes para o campo da preservação patrimonial, apesar de evidenciar que o patrimônio cultural, de fato, tem sido uma preocupação de segunda ordem em projetos voltados à "smartificação" das cidades.

Para a abordagem desses assuntos, o artigo foi organizado da seguinte forma: a segunda seção dedica-se à exposição dos conceitos gerais que orientaram a pesquisa, partindo-se dos campos da preservação patrimonial e das cidades inteligentes. A terceira seção apresenta os procedimentos metodológicos utilizados para a RSL, cujos resultados são apresentados na quarta seção, seguido das considerações finais com uma síntese do que foi discutido.

\section{Conceitos gerais e panorama da literatura}

Para o entendimento do presente estudo, faz-se necessário, inicialmente, contextualizar as duas principais temáticas aqui abordadas: o campo do patrimônio cultural e os recentes estudos sobre as cidades inteligentes, por meio de uma análise de seu desenvolvimento na literatura e dos conceitos gerais que embasam cada uma delas. 


\subsection{O patrimônio cultural}

Até o século XVIII o termo mantinha o caráter de herança particular, sendo este o momento em que assume uma nova significação, ligada à esfera pública, conforme apontam Choay (2001) e Le Goff (2005). De acordo com esses autores, a Revolução Francesa, iniciada em 1789, representou o marco inicial em uma mudança de atitude, com a instituição dos chamados bens da nação: edifícios, monumentos, obras de arte e outros tipos de bens que pudessem difundir valores patrióticos e rememorar a história francesa.

As práticas de preservação foram se especializando e profissionalizando ao longo do século XIX, com a consolidação das primeiras legislações sobre o tema, de âmbito regional, como, por exemplo, a lei francesa sobre monumentos históricos, de 1887. Já no século XX, começaram a ser discutidas as principais normas e recomendações de caráter internacional, especialmente a partir da fundação da Sociedade das Nações, em 1919. Foi por meio dessa organização que foi editada a primeira Carta internacional para a preservação patrimonial, a Cartas de Atenas, de 1931, estabelecendo princípios básicos da conservação e restauração de bens históricos.

A segunda Carta de Atenas, de 1933, já revelava uma das questões fundamentais para a preservação patrimonial: a tensão entre o que deveria ser mantido, com relação ao passado das cidades, e o que deveria desaparecer para permitir a expansão delas. A Carta, fruto das discussões do Congresso Internacional de Arquitetura Moderna (CIAM), colocava a problemática da seguinte forma:

A morte, que não poupa nenhum ser vivo, atinge também as obras dos homens. É necessário saber reconhecer e discriminar nos testemunhos do passado aqueles que ainda estão bem vivos. Nem tudo que é passado tem, por definição, direito à perenidade; convém escolher com sabedoria o que deve ser respeitado. (CIAM, 1933).

A questão do que preservar e do que esquecer constituiu também um dos principais pontos na primeira norma nacional de preservação patrimonial, o Decreto-Lei $n^{\circ} 25$, de 1937, que definia como 
[...] patrimônio histórico e artístico nacional o conjunto dos bens móveis e imóveis existentes no país e cuja conservação seja de interesse público, quer por sua vinculação a fatos memoráveis da história do Brasil, quer por seu excepcional valor arqueológico ou etnográfico, bibliográfico ou artístico. (BRASIL, 1937).

Ao longo das décadas, a noção dos tipos de patrimônio que deveriam ser preservados foi sendo alargada, juntamente com a própria noção de cidadania e de que a preservação da memória de grupos e indivíduos é um direito de todos. Assim, em 1964, foi editada a Carta de Veneza, documento que ampliou as definiçõoes e diretrizes iniciadas na Carta de Atenas, de 1931. Já em 1975, durante a IV Assembleia Geral do Conselho Internacional de Monumentos e Sítios (ICOMOS), foi iniciada a discussão sobre a necessidade de preservação de pequenos centros históricos, em todos os seus aspectos. De acordo com Froner (2014, p. 38),

[...] pela primeira vez não se olha apenas para a materialidade do Patrimônio, para a estrutura dos complexos arquitetônicos ou sítios arqueológicos: a maior preocupação exposta está voltada para a população que vive no entorno ou no próprio centro histórico.

Segundo Castriota (2007, p. 16), com relação ao patrimônio arquitetônico, “[...] a sua concepção inicial, muito presa ainda à ideia tradicional de monumento histórico único, vai sendo ampliada: [...] aos critérios estilísticos e históricos vão se juntando outros, como a preocupação com o entorno, a ambiência e o significado".

Foi nesse momento que, no Brasil, implantou-se o Programa de Cidades Históricas, primeira iniciativa federal voltada para a recuperação do patrimônio cultural urbano e do desenvolvimento econômico local, estimulado principalmente pelo turismo cultural (CORREA, 2016). Em 1975 foi também criado o Centro Nacional de Referência Cultural, que "se voltava para o referenciamento da cultura 'viva', sobretudo daquela enraizada no fazer popular, como forma de tornar mais 'nacional' e mais 'plural' a representação da cultura brasileira." (FONSECA, 1996, p. 156).

Internacionalmente, diversos instrumentos representam marcos das discussões sobre novas temáticas dentro da área patrimonial, tais como a 
Declaração de Estocolmo (1972), que trata sobre o ambiente humano e aborda a questão da sustentabilidade; a Carta do Turismo Cultural (1976); a Declaração do México (1985), sobre políticas culturais; a Carta de Mar del Plata (1997) e a Recomendação de Paris (2003), sobre o patrimônio imaterial. No Brasil, a Constituição Federal de 1988 trouxe um conceito de patrimônio ampliado e flexível, que abarca as esferas material e imaterial.

Percebe-se que as políticas patrimoniais envolvem diferentes interesses, de diferentes setores da sociedade, e sua relação com a dinâmica urbana, especialmente se considerado o patrimônio edificado, será sempre fruto de tensões. Assim, para avaliar de que forma essa área se coloca diante de iniciativas relacionadas às cidades inteligentes, é preciso conhecer um pouco de seu histórico.

\subsection{As cidades inteligentes}

Segundo Hajduk (2016, p. 36), o conceito de smart city começou a ser utilizado ainda na década de 1990. A primeira menção a esse termo encontrada na literatura refere-se a uma conferência realizada em 1990 na cidade de São Francisco, nos Estados Unidos: “Atingindo uma economia global sustentável: infraestruturas conectadas - cidades inteligentes, sistemas rápidos, redes globais" (GIBSON; KOZMETSKY; SMILOR, 1992).

Ao longo dos anos 1990 e 2000, os termos digital cities e smart cities começaram a se popularizar, sendo que, durante a década de 2000, a utilização do termo digital city era predominante na literatura (COCCHIA, 2014, p. 28). A partir de 2010, contudo, as publicações abordando especificamente as smart cities se multiplicaram, devido, principalmente, ao fomento da União Europeia (UE) a essas iniciativas (HAJDUK, 2016).

Autores como Caragliu, Del Bo \& Nijkamp (2011, p. 50) definem da seguinte forma as cidades inteligentes:

Uma cidade é inteligente quando investimentos em capital humano e social e infraestrutura tradicional (transporte) e moderna (TIC) fomentam o crescimento econômico sustentável e uma alta qualidade de vida, com gestão inteligente dos recursos naturais, através de uma governança participativa. 
Já Hollands (2008, p. 305) apresenta uma outra visão: “Territórios com alta capacidade de aprendizado e inovação, que se baseiam na criatividade de sua população, em suas instituições de produção de conhecimento e sua infraestrutura digital para comunicação."

Embora os conceitos não sejam totalmente uniformes, dois pontos podem ser considerados consensuais: que as cidades inteligentes são propostas para se lidar com os problemas atuais, pensando em um modelo de cidade que se almeja para o futuro; e que a operacionalização das cidades inteligentes se dá por meio das tecnologias de informação e comunicação (TIC) existentes e daquelas que ainda podem surgir. Como afirma Arroub et al. (2016, p. 180):

Devido ao fenômeno da urbanização, surgiu um número significativo de riscos, preocupações e problemas. Consequentemente, as últimas administrações têm buscado soluções ótimas, que só podem ser encontradas, de acordo com os pesquisadores, na "inteligência". Mais além, a inteligência pode significar ser sustentável, habitável, seguro, verde ou conectado. De fato, a cidade inteligente pode ser definida como a busca por alcançar todos esses objetivos usando as TICs.

Assim, baseando-se em Giffinger \& Gudrun (2010), Chourabi et al. (2012) e Zubizarreta, Seravalli \& Arrizabalaga (2015), que realizaram uma análise sistêmica de todos os fatores considerados sobre as cidades inteligentes, é possível identificar sete áreas prioritárias contempladas pelas iniciativas inteligentes: economia, pessoas (capital humano), governança, mobilidade, meio ambiente, qualidade de vida e tecnologia.

Com base nesses fatores, Figueiredo (2018) distingue dois tipos diferentes de aplicação do conceito de cidades inteligentes. Em um primeiro momento, no início da década de 2000, empresas de tecnologia e escritórios de arquitetura propuseram a construção de cidades inteiras do zero, "para mostrar que a cidade $100 \%$ sustentável não apenas estaria sob nosso alcance como também teria um excelente padrão de vida" (FIGUEIREDO, 2018, p. 20). Os empreendimentos de Masdar, na Arábia Saudita, e de Songdo, na Coreia do Sul, são os principais exemplos desses projetos, que se mostraram investimentos inviáveis em função da rápida mudança de tecnologias desenvolvidas no 
período. Masdar e Songdo, dessa forma, não chegaram a ser finalizadas, mesmo após um gasto massivo de recursos.

Em um segundo momento, que vai de 2007 a 2014, foram desenvolvidos projetos mais realistas, voltados para a smartificação de cidades reais, com base nas categorias de ação já citadas. A partir de 2014, entretanto, Figueiredo (2018) aponta a inserção da smartificação nas agendas nacionais, por meio de projetos que investem em aplicativos e coleta de dados. No Brasil, programas nesse sentido foram desenvolvidos em nível federal, como o "Minha Cidade Inteligente", e em nível municipal, com a "Rede Brasileira de Cidades Inteligentes e Humanas".

Até o momento, pouca ou nenhuma menção aos aspectos culturais que fazem parte da vida nas cidades foi feita pelos autores consultados, e quando existe, constitui um subtópico da área de qualidade de vida. Já com relação ao patrimônio cultural, apenas um autor o menciona: para Neirotti et al. (2014, p. 27), com relação às aplicações das smart cities "[...] no caso da cultura, o envolvimento do público poderia ter como objetivo melhorar a exploração e atratividade do patrimônio cultural de uma cidade.”.

Percebendo que a preservação patrimonial se encontrava subrepresentada nos trabalhos sobre cidades inteligentes, uma série de autores deu início a um tópico de estudo denominado "patrimônio inteligente", que considera, principalmente, o uso de alternativas tecnológicas para a preservação e difusão do patrimônio cultural. Esse assunto será tratado a partir de agora.

\section{Metodologia}

Para a realização deste estudo, de caráter qualitativo e exploratório, optou-se por uma revisão sistemática de literatura (RSL), uma vez que o objeto desta pesquisa ainda é recente e pouco estruturado. Para tanto, foi construído um protocolo específico de pesquisa, seguindo as etapas elencadas por Morandi \& Camargo (2015):

a) formulação da questão de pesquisa;

b) localização dos estudos necessários;

c) realização da seleção e avaliação dos estudos encontrados; 
d) análise e síntese dos dados coletados.

\section{Questão de pesquisa e definição dos termos de busca}

Buscando elucidar a principal dúvida deste estudo, sobre como a preservação patrimonial seria abordada no contexto das cidades inteligentes, foram realizadas buscas preliminares na base de dados Google Acadêmico, sendo possível identificar que os estudos sobre essa temática não eram abundantes e, mais ainda, que existia uma nomenclatura específica utilizada pelos autores desse campo: o "patrimônio inteligente".

Assim, reduziu-se essa dúvida em termos relacionados entre si por meio de operadores lógicos (AND, OR), adotando a língua inglesa para uma maior recuperação de informações: smart, city (ou cities) e heritage. Nas bases em que foi necessário, os termos foram utilizados também em português e espanhol.

\subsection{Localização dos estudos, seleção e avaliação}

A preservação patrimonial e as cidades inteligentes são, por si, conceitos multidisciplinares, o que foi definido como critério para seleção das bases de dados a serem pesquisadas. Assim, a partir da descrição das bases fornecida pela Coordenação de Aperfeiçoamento de Pessoal de Nível Superior (CAPES), 14 repositórios multidisciplinares ${ }^{3}$ foram selecionados, além da base Google Acadêmico, por possibilitar uma investigação mais ampla.

A pesquisa foi feita em 7 de outubro de 2019, e, por perceber que os resultados recuperados, embora precisos, não representavam um grande número, optou-se por não fazer nenhuma restrição quanto à data ou tipo de publicação, sendo todos os resultados considerados. Essa escolha foi baseada na "regra da exaustividade: uma vez definido o campo do corpus [...], é preciso ter-se em conta todos os elementos desse corpus" (BARDIN, 2011, p. 126).

Foram identificadas 101 referências, que foram exportadas para o gerenciador bibliográfico Zotero, onde foi possível verificar a existência de 45 duplicatas e excluí-las. A partir de então, realizou-se a leitura dos resumos das 56 publicações restantes, sendo todas consideradas pertinentes. 


\subsection{Análise e síntese dos dados}

Para a investigação do corpus documental, foi utilizada a análise de conteúdo, descrita por Bardin (2011). Segundo a autora, esse tipo de abordagem divide-se em uma análise vertical, que se dá no "nível de produção [dos documentos], enquanto variáveis independentes", e uma análise horizontal, a partir do "nível dos textos analisados enquanto variáveis dependentes" (BARDIN, 2011, p. 129).

O primeiro passo foi a definição dos índices de análise, constituídos por temas abordados por cada autor, que seriam de interesse tanto para projetos de cidades inteligentes quanto para a preservação patrimonial. Para viabilizar a exploração do corpus documental, o material foi padronizado no formato PDF e transferido para o software de pesquisa qualitativa NVivo, onde foi criada a estrutura de codificação temática. Após o processo de leitura e com auxílio da ferramenta de frequência de palavras, do software NVivo, foram definidos os índices mais importantes do conjunto documental, estruturando-os em grandes áreas que serão discutidas a partir de agora.

\section{O patrimônio "inteligente"}

Neste capítulo será abordado o "patrimônio inteligente", termo que vem sendo utilizado na literatura internacional para caracterizar os estudos sobre a preservação patrimonial em projetos de cidades inteligentes. Para tanto, serão apontados os aspectos de produção dos trabalhos recuperados na RSL e os assuntos presentes nesses estudos, destacando sua relevância para o conjunto de documentos recuperados.

\subsection{Aspectos de produção}

O termo "patrimônio inteligente", referindo-se a uma abordagem da preservação patrimonial em sintonia com os projetos de cidades inteligentes, começou a aparecer na literatura a partir da década de 2010, sendo que as primeiras tentativas de definição do termo datam de 2013. Trata-se, assim, de uma área recente, conforme apontado no gráfico dos anos de publicação dos documentos, na Figura 1. Na mesma figura, é também possível visualizar os tipos de 
publicações encontradas: 29 artigos (52\%), 20 trabalhos apresentados em conferências (36\%) e sete capítulos de livros (12\%).

Figura 1 - Gráficos de número de publicações por ano e tipos de estudos desenvolvidos com a temática do "patrimônio inteligente"
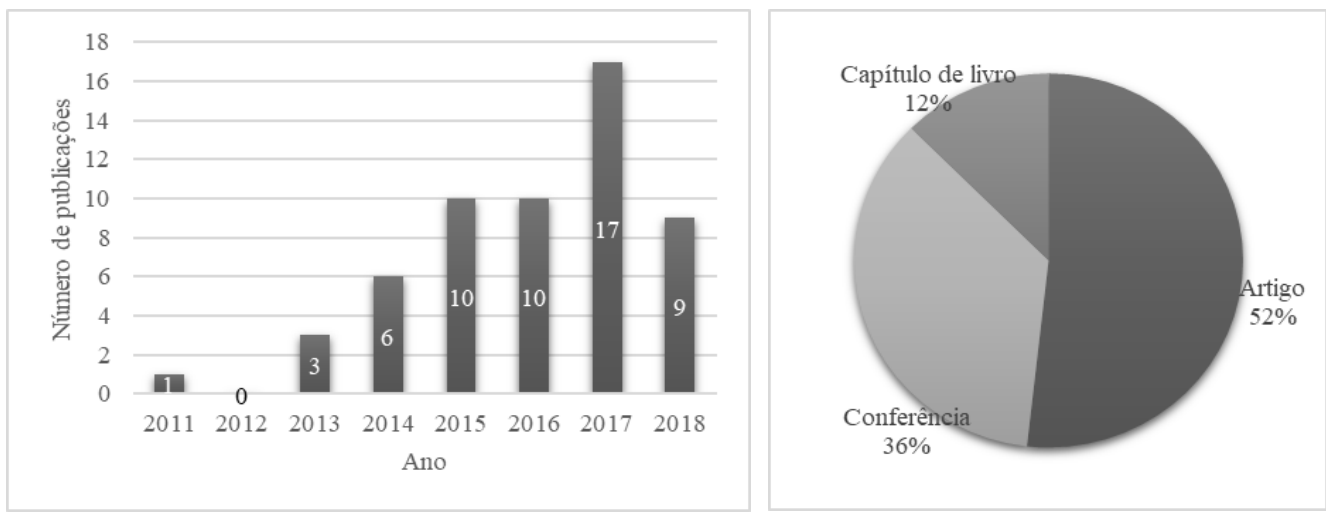

Fonte: Elaborados pela autora com o software de análise qualitativa NVivo.

Com relação aos países de origem dos autores, 12 dos 22 países identificados encontram-se na Europa, sendo a Itália o local onde originam-se o maior número de estudos. Para além da Europa, encontram-se representados a Ásia, em maior proporção, e em menor, a América do Norte, a Oceania e a África, mas não há nenhum trabalho da América Latina, conforme a Tabela 1.

Tabela 1 - Número de publicações sobre a temática do "patrimônio inteligente" por país

\begin{tabular}{|c|c|c|c|c|c|c|c|}
\hline \multirow[b]{3}{*}{ Países } & \multicolumn{7}{|c|}{ Número de publicações } \\
\hline & 1 & 2 & 3 & 4 & 5 & 9 & 22 \\
\hline & $\begin{array}{l}\text { África do Sul, } \\
\text { Bangladesh, Bélgica, } \\
\text { Eslovênia, França, } \\
\text { Holanda, Hungria, } \\
\text { Japão, Portugal, } \\
\text { Romênia, Tailândia }\end{array}$ & $\begin{array}{c}\text { Canadá, } \\
\text { China, } \\
\text { Coréia } \\
\text { do Sul, } \\
\text { Suíça }\end{array}$ & $\begin{array}{l}\text { Austrália, } \\
\text { EUA }\end{array}$ & $\begin{array}{c}\text { Índia, } \\
\text { Inglaterra }\end{array}$ & Grécia & Espanha & Itália \\
\hline
\end{tabular}
Fonte: Elaborado pela autora.

Ainda com relação aos autores, identificou-se que as áreas de atuação concentram-se basicamente em Arquitetura e Engenharia (45\%), Tecnologia da Informação e Engenharia Elétrica (23\%). É importante apontar a falta de pesquisadores de disciplinas que, tradicionalmente, são das mais atuantes nas práticas de preservação patrimonial: enquanto há apenas um autor da área de 
Conservação e Restauração, não há autores das áreas da História e da Museologia. Além disso, a análise das referências bibliográficas dos trabalhos confirma que os três campos acima citados são pouco consultados para o desenvolvimento das pesquisas, sendo predominante o campo da tecnologia.

Complementando a questão da falta de referências multidisciplinares na elaboração desses trabalhos, destaca-se que as publicações fazem poucas referências aos outros trabalhos sobre "patrimônio inteligente" aqui levantados. Não por acaso, 33 dessas publicações nunca foi citada ${ }^{4}, 18$ receberam de uma a três citações, e apenas cinco delas contam um número considerável - de 20 a 50 citações. Para uma área em desenvolvimento, torna-se problemático não tomar conhecimento de estudos similares que estão sendo desenvolvidos, residindo aqui a grande importância de uma revisão sistemática sobre esse tema.

\subsection{Análise de assuntos e definições}

Os assuntos abordados pelos autores dessas publicações foram levantados a partir da análise de conteúdo do corpus documental. Partiu-se da codificação dos tópicos mais tratados, agrupando-os, por semelhança, em grandes áreas, conforme a metodologia indicada na seção 3.

No desenvolvimento dessa análise, percebeu-se que as grandes áreas de interesse para os autores do "patrimônio inteligente" são basicamente as mesmas presentes nos estudos das cidades inteligentes, conforme apontado anteriormente, acrescentando-se somente as ações de preservação patrimonial propriamente ditas, conforme descritas na Tabela 2.

Assim, no campo da Economia, é um consenso entre 28 dos autores $\operatorname{consultados}^{5}$ que o patrimônio histórico e cultural de uma cidade é um ativo econômico, devendo ser utilizado como tal, especialmente por meio do turismo e da economia criativa. Essa diversificação nos usos do patrimônio teria como objetivo final sua sustentabilidade econômica, dependendo cada vez menos de repasses governamentais. Ainda nesse sentido, mas com foco nos edifícios históricos, outros dez autores ${ }^{6}$ defendem a adaptabilidade e o reuso dessas construções como um dos principais pontos para uma política de preservação no âmbito das cidades inteligentes, uma vez que a cidade precisa, cada vez mais, de 
espaços para expansão de sua infraestrutura de serviços. Ambos os assuntos, entretanto, já vêm sendo trabalhados no campo patrimonial desde a década de 1990, não constituindo uma demanda específica das cidades inteligentes.

Tabela 2 - Áreas e tópicos de interesse sobre "patrimônio inteligente" para os autores

\begin{tabular}{ll}
\hline Grandes áreas & Tópicos de interesse \\
\hline Economia & $\begin{array}{l}\text { Patrimônio como ativo; adaptabilidade e reuso; sustentabilidade } \\
\text { econômica; economicidade e eficiência; economia criativa; turismo. }\end{array}$ \\
\hline Pessoas & $\begin{array}{l}\text { Colaboração; integração; participação; representação; novas habilidades } \\
\text { (literacias); privacidade. }\end{array}$ \\
\hline Governança & Descentralização; branding urbano; gentrificação; políticas públicas. \\
\hline Mobilidade & Acessibilidade. \\
\hline Meio ambiente & Sustentabilidade ambiental. \\
\hline Qualidade de vida & $\begin{array}{l}\text { Cidadania; inclusão; diversidade; preservação da memória; identidade e } \\
\text { pertencimento. }\end{array}$ \\
\hline Tecnologia & $\begin{array}{l}\text { Inovação; automatização e análise de dados; visibilidade; interação; } \\
\text { acesso; segurança; amigabilidade e usabilidade; interoperabilidade; } \\
\text { sensores de dados; IoT; Big Data, AR. }\end{array}$ \\
\hline $\begin{array}{l}\text { Ações de } \\
\text { preservação }\end{array}$ & $\begin{array}{l}\text { Paisagens urbanas; cidades e/ou núcleos "históricos"; museus virtuais; } \\
\text { plataformas colaborativas; curadoria distribuída. }\end{array}$ \\
\hline \multicolumn{1}{c}{ Fonte: Elaborado pela autora. }
\end{tabular}

Sobre as Pessoas, 32 autores $^{7}$ ressaltam capacidades como colaboração, integração, participação e representação como fundamentais para a melhoria nos projetos de preservação patrimonial. Entretanto, considera-se que essas temáticas não constituem uma novidade na área patrimonial. Ao contrário, devido à constante limitação de recursos nessa área, as colaborações e integrações entre diferentes instituições e profissionais são uma prática consolidada. Contudo, alguns autores destacam tópicos interessantes, em consonância com questões levantadas por projetos de cidades inteligentes: a necessidade de que as pessoas adquiram novas habilidades para lidar com as inovações tecnológicas que fazem ou farão parte também da área cultural e patrimonial (MARKOVIC; SOFRONIJEVIC, 2015; MCKENNA, 2017) e a discussão sobre o direito à privacidade, levando em conta a pretensão de utilização de sensores de dados para captação automática de dados do público de espaços culturais, visando contribuir com sua conservação preventiva e adaptar os serviços oferecidos à população. Esses temas, portanto, são demandas mais atuais, e que merecem maior espaço de discussão junto à área patrimonial. 
As áreas de Governança, Mobilidade e Meio Ambiente são as menos trabalhadas pelos autores, e todos os tópicos levantados já vêm fazendo parte das discussões patrimoniais nas últimas décadas. Por exemplo, a formulação de políticas públicas que garantam a efetivação da preservação patrimonial e os benefícios de uma gestão descentralizada, com maior poder de decisão aos municípios, temas abordados por 27 autores $^{8}$, são reivindicações antigas no campo patrimonial. Da mesma forma, a utilização do patrimônio cultural apenas para o branding urbano e a consequente gentrificação de áreas tradicionais, apontados por Campos \& Del Marmol (2017), são preocupações de longa data no âmbito patrimonial. A acessibilidade (física e intelectual) e a sustentabilidade, tópicos de 12 autores ${ }^{9}$, são outras temáticas amplamente exploradas junto ao patrimônio histórico e cultural.

Com relação à Qualidade de Vida, mais uma vez os tópicos abordados não constituem novidade. Cidadania, inclusão, diversidade, preservação da memória, identidade e pertencimento, pontos de atenção para outros 27 autores $^{10}$, são termos que podem ser encontrados na maioria das publicações, documentos normativos e manuais da área patrimonial. $\mathrm{O}$ mesmo ocorre com relação às Ações de Preservação, com uma ressalva para a narrativa de Lupo \& Ozdil (2013) sobre os museus virtuais, plataformas colaborativas e curadoria distribuída, temas mais recentes e que ainda estão em desenvolvimento.

O grande diferencial dos trabalhos sobre "patrimônio inteligente" reside mesmo na descrição da área da Tecnologia em proveito da preservação e difusão patrimonial. Isso se dá pelo próprio desenvolvimento do campo tecnológico, que lança novos produtos com espaços de tempo cada vez menores. Novos dispositivos e aplicativos surgem todo dia, e as discussões sobre suas aplicações no campo patrimonial não acompanham totalmente o mesmo ritmo.

Mesmo assim, é importante destacar que, no caso de bens digitalizados ou nato-digitais, eles já são objeto de estudo da área patrimonial desde o início do século XXI, especialmente a partir de 2003, com a realização do Conselho Intergovernamental do Programa "Informação para Todos", da UNESCO, que resultou na publicação da Carta para Preservação do Patrimônio Digital. De acordo com esse documento, recursos digitais fazem parte do acervo de 
conhecimento e expressões dos seres humanos, e requerem diretrizes especiais para sua preservação, já que se tratam de objetos efêmeros:

Os materiais digitais incluem textos, bancos de dados, imagens fixas e em movimento, áudio, gráficos, software e páginas da web, entre uma ampla e crescente variedade de formatos. Eles são frequentemente efêmeros e requerem retenção, produção, manutenção e gerenciamento intencionais. [...] Muitos desses recursos têm valor e significado duradouros e, portanto, constituem um patrimônio que deve ser protegido e preservado para as gerações atuais e futuras (UNESCO, 2003).

A diferença que as publicações aqui analisadas trazem passa pela aplicação de novas tecnologias para a preservação, principalmente, de bens não digitais, sendo que um dos objetivos desses estudos é a própria digitalização desses bens, por meio de novas tecnologias. Assim, dentre os tipos de tecnologia mencionados nos trabalhos que discutem ou demostram sua aplicação, é possível destacar três principais.

A Internet of Things, ou Internet das Coisas ${ }^{11}$ (IoT), que se baseia no uso de sensores e transmissores de dados para conectar, via internet, objetos do uso cotidiano entre si e com o público, e uma das principais aplicações dessa tecnologia seria o monitoramento das condições ambientais de bens edificados. A Augmented Reality, ou Realidade Aumentada ${ }^{12}$ (AR), que adiciona ao mundo "real" uma camada virtual, elaborada por meio de vídeos em $360^{\circ}$ e escaneamentos em três dimensões (3D), e é sugerida para dinamizar a difusão de bens, especialmente para aqueles que não podem visitá-los fisicamente. E as Plataformas Colaborativas ${ }^{13}$, que permitem ao público adicionar e usufruir de dados sobre bens culturais, disponível na web ou em aplicativos para smartphones.

Os assuntos abordados pelos autores ajudam também a compreender as tentativas de conceituação do chamado "patrimônio inteligente". Em 2013, Lupo e Özdil deram o primeiro passo para a criação desse conceito, ao relacionar a preservação do patrimônio com a disponibilização e utilização de informações difusas, sendo necessário torná-las "acessíveis, precisas, visíveis e relacionais" para que a preservação do patrimônio fosse considerada "inteligente" (LUPO; ÖZDIL, 2013, p. 13). Também em 2013, Vattano relacionou o sucesso das 
estratégias de cidades inteligentes "à redescoberta e à melhoria de antigos valores e relações, graças ao apoio de inovações tecnológicas" (2013, p. 269). Sendo assim, o patrimônio cultural seria uma parte essencial das cidades, e o apoio das tecnologias para sua redescoberta e preservação constituiriam uma "atitude patrimonial inteligente".

A partir de 2015 as publicações praticamente duplicaram em relação ao ano anterior. Segundo Sturiale \& Trovato (2015, p. 953), “[...] é possível introduzir o conceito de patrimônio inteligente como o processo de desenvolvimento que pode ser implementado através do uso do conhecimento." Trabalhos como o de Qiu, Li \& Sun (2015, p. 260) abordam uma perspectiva humana do conceito: "O patrimônio não é apenas inteligência técnica, mas também inteligência humana. Isso requer a construção de um patrimônio inteligente e a necessidade de se concentrar nas pessoas e nos serviços.”. Enquanto autores como Riganti e Khoshelham dão maior ênfase ao uso das tecnologias: "Uma agenda para o patrimônio inteligente deve centrar-se no desenvolvimento de infraestruturas de TIC com acesso aberto." (RIGANTI, 2017, p. 23-24) e "O patrimônio inteligente refere-se ao uso de tecnologias para otimizar a tomada de decisões no uso e gestão de edifícios patrimonializados" (KHOSHELHAM, 2018, p. 7). Unindo essas dimensões, destaca-se a definição de Borda \& Bowen (2017) como a que mais se aproxima de caracterizar de fato uma área de atuação junto à preservação patrimonial:

O conceito de "patrimônio cultural inteligente" consiste em conectar digitalmente instituições, visitantes e objetos, em um diálogo. O patrimônio inteligente foca na adoção de abordagens mais participativas e colaborativas, disponibilizando gratuitamente os dados culturais (dados abertos) e, consequentemente, aumentando as oportunidades de interpretação, curadoria digital e inovação (BORDA; BOWEN, 2017, p. 10) (grifos da autora).

Percebe-se, aqui, uma síntese dos assuntos mais abordados pelo corpus documental, indicando que, ao falar do "patrimônio inteligente", é necessária a reflexão sobre formas de possibilitar a conexão virtual das partes envolvidas na gestão patrimonial para, com uso de abordagens colaborativas, disponibilizar o máximo de informações possíveis e fomentar a inovação. 
É importante ressaltar que essa definição indica uma forma de se lidar com o patrimônio cultural, intermediada por ferramentas tecnológicas, não confundindo-se, assim, com o patrimônio digital, que se refere a um tipo diferente de objeto de preservação. Ao mesmo tempo, a abordagem não se opõe, em nenhum momento, ao campo de gestão e preservação do patrimônio cultural, sendo, na verdade, uma complementação a ele. Assim, acredita-se que estudos sobre o "patrimônio inteligente" podem ser de grande valia para engendrar novas formas de preservação, fruição e difusão do patrimônio cultural.

\section{Considerações finais}

Atualmente, no Brasil, uma série de iniciativas "inteligentes" vem sendo realizada, com uma grande ênfase à utilização de tecnologias para tornar digitais vários tipos de serviços. Prova disso é o fato de que algumas cidades brasileiras, tais como Rio de Janeiro, São Paulo e Belo Horizonte, fazem parte de rankings internacionais como o Global Power City Index (Fundação Mori), o Cities in Motion Index (IESE School of Business) e o Global Cities Index (A.T. Kearney). Ao analisar as ações "inteligentes" dessas cidades, porém, encontram-se poucas iniciativas voltadas à área cultural e ao patrimônio cultural.

Os estudos sobre o "patrimônio inteligente" apresentam, assim, o mérito de levantar a questão da importância da preservação patrimonial para as cidades, qualquer que sejam suas estratégias de governança e planejamento para o futuro. Na medida em que as ações smartificadoras já se encontram em plena execução, com ou sem a presença da área cultural em seu escopo, é necessário que as demandas culturais sejam inseridas na arena de tais discussões. Para tanto, aproximar essas demandas da linguagem que vem sendo empregada nos estudos de smart cities constitui uma estratégia válida, embora seja imprescindível que o aporte teórico e metodológico da área patrimonial seja igualmente considerado.

O uso dos diferentes tipos de tecnologias citados nos estudos recuperados é também de grande importância para a área patrimonial, apresentando ganhos reais do ponto de vista da conservação preventiva, da fruição cultural, da difusão e do acesso aos bens culturais. Entretanto, ressalta-se 
que o otimismo com o uso da tecnologia não deve camuflar questões problemáticas, como o direito das pessoas aos seus dados e à privacidade frente ao uso indiscriminado de sensores automáticos. Nem com relação aos graves problemas sociais e econômicos que fazem parte da realidade nacional, que requerem políticas públicas muito mais complexas que o desenvolvimento de um aplicativo.

Com relação ao termo "patrimônio inteligente", é importante relembrar que ele surgiu no âmbito dos estudos sobre cidades inteligentes, e por isso herdou uma parte de seu nome. A utilização da palavra "inteligente" não foi percebida, em nenhum dos trabalhos analisados, como uma oposição ou uma hierarquização com relação às demais áreas de preservação patrimonial. Mesmo assim, optou-se por usar o termo sempre entre aspas, para evitar esse tipo de interpretação.

Independente do nome utilizado, percebe-se que esses estudos apresentam novas possibilidades para a área patrimonial, devendo sempre concorrer para que as mais diferentes camadas da população brasileira possam, efetivamente, usufruir e ser representadas pelo patrimônio nacional.

\section{Referências}

ANGELIDOU, M. et al. Cultural heritage in smart city environments. International Archives of the Photogrammetry, Remote Sensing \& Spatial Information Sciences, v. 42, p. 27-32, 2017.

APRÓ, D. et al. Can Smart City tools support historical cities become more resilient and regenerative? In: 32TH INTERNATIONAL CONFERENCE ON PASSIVE AND LOW ENERGY ARCHITECTURE, 2016, Coruña. Anais... Coruña: [s.n.], 2016.

ARDELEANU, A.; PAVĂL, O. Industrial heritage in the smart city context. Scientific Bulletin of the Petru Maior University of Tîrgu Mureş, v. 13, n. 2, p. 43-46, 2016.

ARROUB, A. et al. A literature review on Smart Cities: Paradigms, opportunities and open problems. In: 2016 INTERNATIONAL CONFERENCE ON WIRELESS NETWORKS AND MOBILE COMMUNICATIONS, 2016, [S.1.]: IEEE, 2016. p. 180-186. 
BAEZA, F. J. et al. Structural health monitoring systems for smart heritage and infrastructures in Spain. Mechatronics for cultural heritage and civil engineering, v. 92, p. 271-294, 2018.

BARDIN, L. Análise de conteúdo. São Paulo: Edições 70, 2011.

BONACINI, E. \# iziTRAVELSicilia, a participatory storytelling project/process: bottom-up involvement of Smart Heritage communities. International Journal of Interactive Communication Systems and Technologies, v. 7, n. 2, p. 24-52, 2017.

BORDA, A.; BOWEN, J. Smart cities and cultural heritage: a review of developments and future opportunities. In: EVA LONDON 2017:

ELECTRONIC VISUALISATION AND THE ARTS, 2017, Londres. Anais... Londres: BCS Learning \& Development Ltd., 2017. p. 9-18.

BRASIL. Decreto-lei n ${ }^{\circ} 25$, de 30 de novembro de 1937. Organiza a proteção do patrimônio histórico e artístico nacional. Brasília, DF: Presidência da República, 1937. Disponível em: <http://www.planalto.gov.br/ccivil_03/DecretoLei/Del0025.htm>. Acesso em: 4 nov. 2019.

BRUSAPORCI, S.; CENTOFANTI, M.; MAIEZZA, P. MUS.AQ: a digital museum of L'Aquila for the Smart City INCIPICT project. In: INTERNATIONAL CONFERENCE HERITAGEBOT, 2017, Cassino, Italy. Anais... Cassino, Italy: Springer, 2017. p. 200-207.

BURKE, P. Cultura popular na idade moderna: Europa, 1500-1800. São Paulo: Editora Companhia das Letras, 2010.

CAMPOS, B. S.; DEL MARMOL, C. Smart Cities and charming villages: new heritage processes in the twenty-first century. Revista de Dialectologia $Y$ Tradiciones Populares, v. 72, n. 2, p. 359-377, dez. 2017.

CARAGLIU, A.; DEL BO, C.; NIJKAMP, P. Smart cities in Europe. Journal of Urban Technology, v. 18, n. 2, p. 65-82, 2011.

CASTRIOTA, L. B. Intervenções sobre o patrimônio urbano: modelos e perspectivas. Fórum Patrimônio: ambiente construído e patrimônio sustentável, v. 1, n. 1, dez. 2007. 
CERASOLI, M. The recovery of small historical centres, toward "historical small smart cities". Architecture, City and Environment, v. 11, n. 33, p. 155$180,2017$.

CHIANESE, A.; PICCIALLI, F. A smart system to manage the context evolution in the Cultural Heritage domain. Computers \& Electrical Engineering, v. 55, n. C, p. 27-38, 2016.

CHOAY, F. A alegoria do patrimônio. São Paulo: Estação Liberdade; Editora UNESP, 2001.

CHOURABI, H. et al. Understanding smart cities: an integrative framework. In: 45TH HAWAII INTERNATIONAL CONFERENCE ON SYSTEM

SCIENCES, 2012, Hawaii. Anais... Hawaii: IEEE, 2012. p. 2289-2297.

CIAM, C. I. DE A. M. Carta de Atenas de 1933. . [S.1.]: IPHAN. Disponível em:

<http://portal.iphan.gov.br/uploads/ckfinder/arquivos/Carta\%20de\%20Atenas\% 201933.pdf>. Acesso em: 4 nov. 2019. , 1933

COCCHIA, A. Smart and digital city: a systematic literature review. In: Smart city. Progress in IS. Cham: Springer, 2014. p. 13-43.

CORREA, S. M. O Programa de Cidades Históricas: por uma política integrada de preservação do patrimônio cultural urbano. Anais do Museu Paulista: História e Cultura Material, v. 24, n. 1, p. 15-58, abr. 2016.

COUNSELL, J. The Potential of Living Labs for Smart Heritage Building Adaptation. Advanced Technologies for Sustainable Systems. Cham: Springer, 2017. p. 41-50.

DE MASI, A. Smart cultural heritage and open source as regeneration of historical centers. In: 4TH INTERNATIONAL CONFERENCE ON BIG DATA AND CLOUD COMPUTING, 2014, Sydney. Anais... Sydney: IEEE, 2014. p. 729-736.

DI NATALE, I.; GENTILE, A. Smart Heritage: Cultural Heritage Experiences Project through Mobile Technology, Augmented Reality, Cultural Tourism and Digital Storytelling. In: XIV INTERNATIONAL FORUM WORLD 
HERITAGE AND DEGRADATION: SMART DESIGN, PLANNING AND TECHNOLOGIES, 2016, Napoles, Capri. Anais... Napoles, Capri: [s.n.], 2016.

DIEGO, F. J.; ESTEBAN, B.; MERELLO, P. Design of a hybrid (wired/wireless) acquisition data system for monitoring of cultural heritage physical parameters in Smart Cities. Sensors, v. 15, n. 4, p. 7246-7266, abr. 2015.

ENTRECANALES, R. R. Smart Heritage City (SHCity) en el Año Europeo del Patrimonio: un proyecto de cooperación europea que busca la gestión global de conjuntos históricos utilizando el desarrollo de nuevas tecnologías aplicadas al patrimonio. Patrimonio histórico de Castilla y León, n. 65, p. 24-27, 2018.

FIGUEIREDO, G. M. P. O discurso e a prática da smart city: perspectivas críticas e aproximações sistemáticas no contexto de metrópoles latinoamericanas. 2018. 156 f. Dissertação de Mestrado - Universidade de São Paulo, São Paulo, 2018.

FONSECA, M. C. L. Da modernização à participação: a política federal de preservação nos anos 70 e 80. Revista do Patrimônio Histórico e Artístico Nacional, v. 24, p. 153-163, 1996.

FRONER, Y. A. Preservação e memória: a ação do Patrimônio Cultural no processo de construção da cidadania. Patrimonium, v. 1, p. 37-41, 2014.

GAMBARDELLA, C. et al. Pompei Knowledge Factory: cultural heritage and ICT for a smart city. In: 2014 INTERNATIONAL CONFERENCE ON VIRTUAL SYSTEMS AND MULTIMEDIA, 2014, Hong Kong. Anais... Hong Kong: IEEE, 2014. p. 132-139.

GIBSON, D. V.; KOZMETSKY, G.; SMILOR, R. W. The technopolis phenomenon: smart cities, fast systems, global networks. Maryland, USA: Rowman \& Littlefield Publishers, 1992. (International Series on Technical Innovation and Entrepreneurship).

GIFFINGER, R.; GUDRUN, H. Smart cities ranking: an effective instrument for the positioning of the cities? ACE: architecture, city and environment, v. 4, n. 12, p. 7-26, 2010. 
GIRARD, L. F. Toward a smart sustainable development of port cities/areas: the role of the "Historic Urban Landscape" approach. Sustainability, v. 5, n. 10, p. 4329-4348, 2013.

GOEL, P. Cultural Heritage, urban planning and the Smart City in indian context. International Journal of Science, Engineering and Technology, v. 9, n. $11,2015$.

HAJDUK, S. The concept of a Smart City in urban management. Business, Management and Education, v. 14, n. 1, p. 34-49, 2016.

HOLLANDS, R. G. Will the real smart city please stand up? Intelligent, progressive or entrepreneurial? City, v. 12, n. 3, p. 303-320, 2008.

JARA, A. J. et al. Internet of Things for cultural heritage of smart cities and smart regions. In: 29TH INTERNATIONAL CONFERENCE ON. ADVANCED INFORMATION NETWORKING AND APPLICATIONS WORKSHOPS, 2015, Gwangju, Korea. Anais... Gwangju, Korea: IEEE, 2015. p. 668-675.

KHOSHELHAM, K. Smart Heritage: challenges in digitisation and spatial information modelling of historical buildings. In: 2ND WORKSHOP ON COMPUTING TECHNIQUES FOR SPATIO-TEMPORAL DATA IN ARCHAEOLOGY AND CULTURAL HERITAGE, 2018, Melbourne, Australia. Anais... Melbourne, Australia: [s.n.], 2018. p. 7-12.

KIM, B. et al. Agatha: predicting daily activities from place visit history for activity-aware mobile services in smart cities. International Journal of Distributed Sensor Networks, v. 11, n. 12, p. 1-12, 2015.

KOUKOPOULOS, Z.; KOUKOPOULOS, D. A participatory digital platform for cultural heritage within smart city environments. In: $12 \mathrm{TH}$ INTERNATIONAL CONFERENCE ON SIGNAL-IMAGE TECHNOLOGY \& INTERNET-BASED SYSTEMS, 2016, Napoles. Anais... Napoles: IEEE, 2016. p. $412-419$.

KOUKOPOULOS, Z.; KOUKOPOULOS, D.; JUNG, J. J. A trustworthy multimedia participatory platform for cultural heritage management in smart city environments. Multimedia Tools and Applications, v. 76, n. 24, p. 2594325981, dez. 2017. 
KULKARNI, S.; DHANAMJAYA, M. Smart libraries for smart cities: a historic opportunity for quality public libraries in India. Library Hi Tech News, v. 34, n. 8, p. 26-30, 2017.

LE GOFF, J. História e memória. 5. ed. Campinas: UNICAMP, 2005.

LIM, V. et al. PLUGGY: A pluggable social platform for cultural heritage awareness and participation. In: Advances in Digital Cultural Heritage. [S.1.]: Springer, 2018. p. 117-129.

LOSAVIO, M.; ROGERS, C.; ELMAGHRABY, A. Digital heritage from the Smart City and the Internet of Things: History or stasis? In: DIGITAL HERITAGE INTERNATIONAL CONGRESS, 2015, Granada, Espanha. Anais... Granada, Espanha: IEEE, 2015. p. 431-434.

LUPO, E.; ÖZDIL, E. Towards a Smart Heritage as future diffused museums: design and communication technologies to innovate the experience of the cultural patrimony in Smart Cities. International Journal of the Inclusive Museum, v. 6, n. 1, p. 159-169, 2013.

MAR, A. et al. An application to improve Smart Heritage City experience. In: Advances in Digital Cultural Heritage. [S.1.]: Springer, 2018. v. 10754. p. 89103.

MARKOVIC, L.; SOFRONIJEVIC, A. Building a Gamified system for caputring MOOC related data: smart city learning community as its most precious source of intangible cultural heritage. In: INTERNATIONAL CONFERENCE ON CULTURE AND COMPUTING, 2015, Kyoto, Japan. Anais... Kyoto, Japan: IEEE, 2015. p. 175-182.

MARSELLA, S.; MARZOLI, M. Smart cities and cultural heritage. In: 14TH INTERNATIONAL CONFERENCE NETWORKING, SENSING AND CONTROL, 2017, Calabria, Italy. Anais... Calabria, Italy: IEEE, 2017. p. 281286.

MCKENNA, H. P. Adaptive Reuse of Cultural Heritage Elements and Fragments in Public Spaces: The Internet of Cultural Things and Applications as Infrastructures for Learning in Smart Cities. In: 13TH INTERNATIONAL CONFERENCE ON SIGNAL-IMAGE TECHNOLOGY \& INTERNETBASED SYSTEMS, 2017, Jaipur, India. Anais... Jaipur, India: IEEE, 2017. p. 479-484. 
MINNER, J. et al. A Smart City Remembers Its Past: Citizens as Sensors in Survey and Mapping of Historic Places. In: Crowdsourcing: Concepts, Methodologies, Tools, and Applications. [S.1.]: IGI Global, 2018. p. 489-516.

MORANDI, M. I. W. M.; CAMARGO, L. F. R. Revisão sistemática da literatura. In: Design science research: método de pesquisa para avanço da ciência e tecnologia. Porto Alegre: Bookman, 2015. p. 141-175.

MOUSHEIMISH, R. et al. Smart preserving of cultural heritage with PACTART. Multimedia Tools and Applications, v. 76, n. 24, p. 26077-26101, 2017.

NARDI, L. Smart Heritage: Technology for marking Architecture. In: XIV INTERNATIONAL FORUM WORLD HERITAGE AND DEGRADATION: SMART DESIGN, PLANNING AND TECHNOLOGIES, 2016, Napoles, Capri. Anais... Napoles, Capri: [s.n.], 2016.

NEIROTTI, P. et al. Current trends in Smart City initiatives: some stylised facts. Cities, v. 38, p. 25-36, 2014.

NERNGCHAMNONG, K. et al. World heritage city surveillance system by a smart CCTV system. Procedia Engineering, v. 8, p. 321-327, 2011.

NEVE, M. Would urban cultural heritage be smart? Culture as a land factor and Italian cities smartness. Revista de Comunicação e Linguagens, n. 48, p. 163190, 2018.

OLIVA, A. G. et al. Smart tourism destination \& cultural heritage: a new unexplorer sector in smart cities develop. International Journal of Scientific Management and Tourism, v. 3, n. 1, p. 389-411, 2017.

QIU, J.; LI, J.; SUN, H. Innovative and applied research on big data platforms of smart heritage. ISPRS Annals of the Photogrammetry, Remote Sensing and Spatial Information Sciences, v. 2, n. 5, p. 257-261, 2015.

QUIST, W. J. et al. Bilateral collaboration in built heritage material research and resource maintenance supportive to smart and sustainable cities. In:

SMART \& SUSTAINABLE CITIES AND TRANSPORT SEMINAR, 2017, Pretoria, South Africa. Anais... Pretoria, South Africa: [s.n.], 2017. p. 59-68. 
RADEJ, B.; PIRKOVIČ, J.; PAQUET, P. Smart heritage policy. Innovative Issues and Approaches in Social Sciences, v. 11, n. 1, p. 57-72, 2018.

RAHAMAN, H. et al. Synchronous location-aware media and augmented visualization for real world tourist (SMART): An application for Khalifatabad heritage site, Bagerhat, Bangladesh. In: 22ND INTERNATIONAL CONFERENCE ON VIRTUAL SYSTEM \& MULTIMEDIA, 2016, Kuala Lumpur, Malaysia. Anais... Kuala Lumpur, Malaysia: IEEE, 2016. p. 1-7.

RIGANTI, P. Smart cities and heritage conservation: developing a smartheritage agenda for sustainable inclusive communities. International Journal of Architectural Research, v. 11, n. 3, p. 16-27, 2017.

SEPE, M. Improving sustainable enhancement of cultural heritage: Smart placemaking for experiential paths in Pompeii. International Journal of Sustainable Development and Planning, v. 10, n. 5, p. 713-733, 2015.

SINGH, R. P. B.; RANA, P. S. Varanasi: sustainable development goals, smart city vision and inclusive heritage development. Kashi Journal of Social Sciences, v. 7, n. 1-2, p. 219-236, dez. 2017.

STAVRINOUDIS, T.; PAPATHANASIOU-ZUHRT, D. Design and delivery of experience-based tourism products and services in heritage settings: the PEGA Training Programme. In: Caring and Sharing: The Cultural Heritage Environment as an Agent for Change. Springer Proceedings in Business and Economics. Cham: Springer, 2018. p. 165-181.

STURIALE, L.; TROVATO, M. R. ICTs and smart territories: the knowledge and use of the UNESCO heritage by using the QR codes system. In: 7TH INTERNATIONAL CONFERENCE ON INFORMATION AND COMMUNICATION TECHNOLOGIES IN AGRICULTURE, FOOD AND ENVIRONMENT, 2015, Kavala, Greece. Anais... Kavala, Greece: [s.n.], 2015. p. 946-956.

TORRALBA, L. T.; HERNÁNDEZ, M. G. Territorios y ciudades inteligentes: retos para convertirse en Smart Heritage Destination. In: XI CONGRESO INTERNACIONAL DE TURISMO Y TECNOLOGIAS DE LA INFORMACION E LA COMUNICACION, 2016, Málaga, Espanha. Anais... Málaga, Espanha: Universidad de Málaga, 2016. p. 109-122. 
TROVATO, M. R.; STURIALE, L. The smart management and the e-cultural marketing of UNESCO heritage. International Journal of Sustainable Agricultural Management and Informatics, v. 2, n. 2-4, p. 155-173, 2016.

UNESCO. Charter on the Preservation of Digital Heritage. . [S.1: s.n.]. Disponível em: <http://portal.unesco.org/en/ev.php-

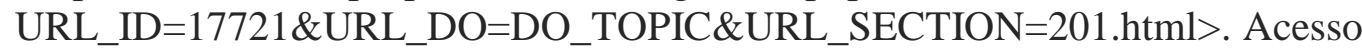
em: 4 nov. 2019.

UPADHYAYA, V. Transforming built heritage towards smart buildings: a case of Walled City Jaipur, Rajasthan. International Journal of Science and Research, v. 6, n. 6, p. 1910-1921, jun. 2017.

VANOLO, A. The image of the creative city, eight years later: Turin, urban branding and the economic crisis taboo. Cities, v. 46, p. 1-7, 2015.

VATTANO, S. Intangible smart city inside tangible historic city: smart heritage as regeneration of Euro-Mediterranean realities. In: 6TH INTERNATIONAL CONGRESS ON SCIENCE AND TECHNOLOGY FOR THE SAFEGUARD OF CULTURAL HERITAGE IN THE MEDITERRANEAN BASIN, 2014a, Rome, Italy. Anais... Rome, Italy: Valmar, 2014. p. 91-100.

VATTANO, S. Smart heritage: un approccio multiscalare. In: La Tecnologia dell'architettura in rete per l'innovazione. Santarcangelo di Romagna: Maggioli Editore, 2016. p. 131-141.

VATTANO, S. Smart Technology for smart regeneration of cultural heritage: Italian smart cities in comparison. In: MUSEUMS AND THE WEB FLORENCE 2014, 2014b, Florence, Italy. Anais... Florence, Italy: [s.n.], 2014.

VATTANO, S. Una rigenerazione smart per i centri storici: da Smart Cities a Smart Heritage. In: Recupero, Valorizzazione, Manutenzione nei Centri Storici: Un tavolo di confronto interdisciplinare. Siracusa: Lettera Ventidue Edizioni, 2013. p. 266-269.

VATTANO, S.; BUJALANCE, S. G. Configuración smart para el patrimonio euro-mediterráneo: el dibujo smart para el Rabato de Agrigento. In: $2^{\text {a }}$ BIENAL DE PROYECTOS DE EDIFICACIÓN Y URBANISMO SOSTENIBLE, 2014, Málaga, Espanha. Anais... Málaga, Espanha: [s.n.], 2014. p. 381-404. 
VOLLARO, R. L. et al. An integrated approach for an historical buildings energy analysis in a smart cities perspective. Energy Procedia, v. 45, p. 372378, 2014.

ZUBIZARRETA, I.; SERAVALLI, A.; ARRIZABALAGA, S. Smart city concept: what it is and what it should be. Journal of Urban Planning and Development, v. 142, n. 1, p. 04015005/1-8, 2015.

ZUMEL, C. M. A un paso del patrimonio inteligente. Patrimonio histórico de Castilla y León, n. 54, p. 41-43, 2015.

\title{
Cultural heritage in smart cities
}

\begin{abstract}
This article aims to investigate the theme of heritage preservation in the context of smart cities. Smart cities are a relatively recent proposal, which first studies date back to the 1990s, begining to be widely propagated since the 2010s. These proposals aim to transform the urban space and its services in a "smart" way, with the use of new information and communication technologies (ICTs), in fields such as health, mobility, security, among others. However, almost nothing is proposed about the cultural heritage preservation, problem which motivated the present study. As methodological approach, a systematic literature review (SLR) was carried out, with 56 publications from different countries, a set that reveals some important issues for the heritage field regarding city "smartification" projects, such as those already taking place in Brazil. As conclusions, is pointed out the proposal of these studies in constitute a new form of heritage preservation - the "smart heritage", discussing its implications, limitations and possibilities.
\end{abstract}

Keywords: Cultural heritage. Preservation. Conservation. Smart cities. Smart heritage.

Recebido: $17 / 12 / 2019$

Aceito: 30/04/2020

\section{Como citar}

CASTRO, Juliana Martins de; BARACHO, Renata Maria Abrantes. O patrimônio cultural nas cidades inteligentes. Em Questão, Porto Alegre, v. 26, n. 3, p. 298-326, set./dez. 2020. DOI: https://doi.org/10.19132/18085245263.298-326. 
${ }^{1}$ Buscas realizadas no Google Acadêmico em 05/09/2019, somente no contexto nacional.

${ }^{2}$ Recuperação dos termos "cidades inteligentes" e "práticas OR manifestações OR equipamentos culturais": 179 resultados. Recuperação dos termos "cidades inteligentes" e "patrimônio cultural": 240 resultados. Buscas realizadas no Google Acadêmico em 05/09/2019, somente no contexto nacional.

${ }^{3}$ As bases selecionadas foram: Cambridge Core, CrossRef Search, EBSCO, Latindex, Oxford Journals, ROAD Directory of Open Access Scholarly Resources, Scielo, Science AAAS, Science Direct e SCOPUS (Elsevier), Springer Link, Wiley Online Library, WorldCat e Web of Science.

${ }^{4}$ Número de citações consultado no Google Acadêmico.

5 São eles: (ANGELIDOU et al., 2017), (ANGELIDOU et al., 2017; APRÓ et al., 2016; BONACINI, 2017; BORDA; BOWEN, 2017; BRUSAPORCI; CENTOFANTI; MAIEZZA, 2017; CAMPOS; DEL MARMOL, 2017; CERASOLI, 2017; DE MASI, 2014; GAMBARDELLA et al., 2014; GIRARD, 2013; JARA et al., 2015; KOUKOPOULOS; KOUKOPOULOS; JUNG, 2017; LIM et al., 2018; LOSAVIO; ROGERS; ELMAGHRABY, 2015; MAR et al., 2018; MCKENNA, 2017; NEVE, 2018; OLIVA et al., 2017; QUIST et al., 2017; RIGANTI, 2017; SEPE, 2015; SINGH; RANA, 2017; STURIALE; TROVATO, 2015; TORRALBA; HERNÁNDEZ, 2016; VANOLO, 2015; VATTANO, 2013, 2014b, 2016).

${ }^{6}$ Os autores: (CERASOLI, 2017; COUNSELL, 2017; GIRARD, 2013; JARA et al., 2015; MINNER et al., 2018; NARDI, 2016; NEVE, 2018; UPADHYAYA, 2017; VATTANO, 2014a; VATTANO; BUJALANCE, 2014).

${ }^{7}$ São eles: (ANGELIDOU et al., 2017; ARDELEANU; PAVĂL, 2016; BONACINI, 2017; BORDA; BOWEN, 2017; BRUSAPORCI; CENTOFANTI; MAIEZZA, 2017; CERASOLI, 2017; CHIANESE; PICCIALLI, 2016; COUNSELL, 2017; DE MASI, 2014; DIEGO; ESTEBAN; MERELLO, 2015; GAMBARDELLA et al., 2014; GIRARD, 2013; GOEL, 2015; JARA et al., 2015; KOUKOPOULOS; KOUKOPOULOS, 2016; KOUKOPOULOS; KOUKOPOULOS; JUNG, 2017; KULKARNI; DHANAMJAYA, 2017; LIM et al., 2018; MAR et al., 2018; MARSELLA; MARZOLI, 2017; MCKENNA, 2017; MINNER et al., 2018; MOUSHEIMISH et al., 2017; OLIVA et al., 2017; QUIST et al., 2017; RIGANTI, 2017; SINGH; RANA, 2017; STURIALE; TROVATO, 2015; VANOLO, 2015; VATTANO, 2014a, b).

${ }^{8}$ Os autores são: (ANGELIDOU et al., 2017; APRÓ et al., 2016; ARDELEANU; PAVĂL, 2016; BORDA; BOWEN, 2017; BRUSAPORCI; CENTOFANTI; MAIEZZA, 2017; CERASOLI, 2017; CHIANESE; PICCIALLI, 2016; DE MASI, 2014; DIEGO; ESTEBAN; MERELLO, 2015; ENTRECANALES, 2018; GAMBARDELLA et al., 2014; GIRARD, 2013; GOEL, 2015; JARA et al., 2015; KIM et al., 2015; KOUKOPOULOS; KOUKOPOULOS, 2016; KOUKOPOULOS; KOUKOPOULOS; JUNG, 2017; LIM et al., 2018; MAR et al., 2018; MARSELLA; MARZOLI, 2017; MINNER et al., 2018; MOUSHEIMISH et al., 2017; NERNGCHAMNONG et al., 2011; RADEJ; PIRKOVIČ; PAQUET, 2018; VATTANO, 2013, 2014b; ZUMEL, 2015).

9 Os seguintes autores: (ANGELIDOU et al., 2017; APRÓ et al., 2016; ARDELEANU; PAVĂL, 2016; BORDA; BOWEN, 2017; DE MASI, 2014; GAMBARDELLA et al., 2014; JARA et al., 2015; KOUKOPOULOS; KOUKOPOULOS; JUNG, 2017; LIM et al., 2018; MINNER et al., 2018; SINGH; RANA, 2017; VATTANO, 2014b).

${ }^{10}$ São eles: (ANGELIDOU et al., 2017; APRÓ et al., 2016; BONACINI, 2017; BORDA; BOWEN, 2017; BRUSAPORCI; CENTOFANTI; MAIEZZA, 2017; CAMPOS; DEL MARMOL, 2017; CERASOLI, 2017; DE MASI, 2014; GAMBARDELLA et al., 2014; GIRARD, 2013; JARA et al., 2015; KOUKOPOULOS; KOUKOPOULOS; JUNG, 2017; LIM et al., 2018; LOSAVIO; ROGERS; ELMAGHRABY, 2015; MAR et al., 2018; MCKENNA, 2017; NEVE, 2018; OLIVA et al., 2017; QUIST et al., 2017; RIGANTI, 2017; SEPE, 2015; SINGH; RANA, 2017; STURIALE; TROVATO, 2015; VANOLO, 2015; VATTANO, 2013, 2014b, 2016). 
${ }^{11}$ Os autores que abordam a IoT são: (BAEZA et al., 2018; CHIANESE; PICCIALLI, 2016; JARA et al., 2015; KOUKOPOULOS; KOUKOPOULOS, 2016; KOUKOPOULOS; KOUKOPOULOS; JUNG, 2017; MARSELLA; MARZOLI, 2017; MCKENNA, 2017; MOUSHEIMISH et al., 2017; OLIVA et al., 2017; STURIALE; TROVATO, 2015; VOLLARO et al., 2014).

${ }^{12}$ Os autores que trabalham com a AR são: (BRUSAPORCI; CENTOFANTI; MAIEZZA, 2017; DE MASI, 2014; DI NATALE; GENTILE, 2016; KHOSHELHAM, 2018; KOUKOPOULOS; KOUKOPOULOS, 2016; KOUKOPOULOS; KOUKOPOULOS; JUNG, 2017; LIM et al., 2018; MCKENNA, 2017; MINNER et al., 2018; QIU; LI; SUN, 2015; RAHAMAN et al., 2016; SEPE, 2015; SINGH; RANA, 2017; VATTANO, 2014b).

13 Plataformas colaborativas são trabalhados por: (BONACINI, 2017; DE MASI, 2014; KOUKOPOULOS; KOUKOPOULOS, 2016; KOUKOPOULOS; KOUKOPOULOS; JUNG, 2017; LIM et al., 2018; STAVRINOUDIS; PAPATHANASIOU-ZUHRT, 2018; STURIALE; TROVATO, 2015; TROVATO; STURIALE, 2016). 